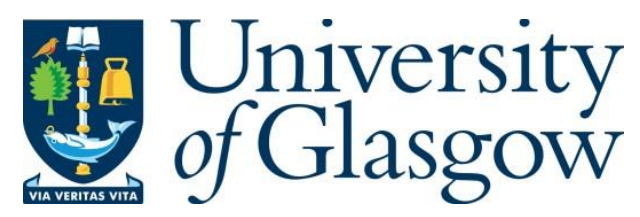

García Núñez, C., Braña, A. F., López, N. and García, B. J. (2018) A novel growth method to improve the quality of GaAs nanowires grown by Ga-assisted chemical beam epitaxy. Nano Letters, 18(6), pp. 3608-3615.

There may be differences between this version and the published version. You are advised to consult the publisher's version if you wish to cite from it.

http://eprints.gla.ac.uk/162504/

Deposited on: 2 October 2018

Enlighten - Research publications by members of the University of Glasgow http://eprints.gla.ac.uk 


\section{A Novel Growth Method to Improve the Quality of \\ GaAs Nanowires Grown by Ga-Assisted Chemical \\ Beam Epitaxy}

Carlos García Núñez, ${ }^{\dagger}$ Alejandro F. Braña, Nair López, Basilio J. García*

Electronics and Semiconductors Group (ELySE), Department of Applied Physics, Universidad Autónoma de Madrid, 28049 Madrid, Spain.

Keywords: GaAs Nanowires, Vapour-Solid-Liquid, Chemical Beam Epitaxy, Ga-assisted Growth, Nanowires Growth on Silicon, Vertically-aligned Nanowires 


\begin{abstract}
The successful synthesis of high crystalline quality and high aspect ratio GaAs nanowires (NWs) with a uniform diameter is needed to develop advanced applications beyond the limits established by thin film and bulk material properties. Vertically aligned GaAs NWs have been extensively grown by Ga-assisted vapour-liquid-solid (VLS) mechanism on $\mathrm{Si}(111)$ substrates, and they have been used as building blocks in photovoltaics, optoelectronics, electronics, etc. However, the nucleation of parasitic species such as traces and nanocrystals on the Si substrate surface during the NW growth could affect significantly the controlled nucleation of those NWs, and therefore the resulting performance of NWs based devices. Preventing the nucleation of parasitic species on the Si substrate is a matter of interest, because they could act as traps for gaseous precursors and/or chemical elements during VLS growth, drastically reducing the maximum length of grown NWs, affecting their morphology and structure, and reducing the NW density along the Si substrate surface.
\end{abstract}

This work presents a novel and easy to develop growth method (i.e. without using advanced nanolithography techniques) to prevent the nucleation of parasitic species, while preserving the quality of GaAs NWs even for long duration growths. GaAs NWs are grown by Ga-assisted chemical beam epitaxy on oxidized $\mathrm{Si}(111)$ substrates using triethylgallium and tertiarybutylarsine precursors by a two-steps based growth method presented here; this method includes a growth interruption for an oxidation on air between both steps of growth, reducing the nucleation of parasitic crystals on the thicker $\mathrm{SiO}_{\mathrm{x}}$ capping layer during the second and longer growth step. VLS conditions are preserved overtime, resulting in a stable NW growth rate of around $6 \mu \mathrm{m} / \mathrm{h}$ for growth times up to $1 \mathrm{~h}$. Resulting GaAs NWs have a high aspect ratio of 85 and average radius of 
$35 \mathrm{~nm}$. We also report for the existence of characteristic reflection high-energy electron diffraction patterns associated to the epitaxial growth of GaAs NWs on $\mathrm{Si}(111)$ substrates, which have been analyzed and compared to the morphological characterization of GaAs NWs grown for different times under different conditions.

\section{Introduction}

Semiconductor nanowires (NWs) have demonstrated their validity and applicability as building blocks in advanced applications such as electronics, optoelectronics, photonics, photovoltaics and sensing, ${ }^{1-6}$ overcoming functionalities and features achieved by thin film and bulk materials, due to their unique properties such as high surface-to-volume ratio, low dimensionality and high crystal quality (i.e. low defect density). Some examples in which GaAs NWs are expected to play a crucial role for the development of a wide number of high-performance applications include photovoltaic cells, ${ }^{7}$ photodetectors, ${ }^{8}$ resonant tunneling diodes, ${ }^{9}$ single electron transistors, ${ }^{10}$ and single photon emitters, ${ }^{11}$ mainly based on their electronic transport properties, such as direct bandgaps and high carrier mobility. Currently available technology to integrate NWs on foreign substrates ${ }^{6}$ or to develop electronic devices directly from as-grown $\mathrm{NWs}^{12}$ presents still a poor performance; both approaches to develop NW-based electronic devices require a synthesis procedure capable to control the resulting density of NWs per unit area, NW aspect ratio (length to diameter ratio, $L_{\mathrm{NW}} / d_{\mathrm{NW}}$ ), NW morphology and NW crystal structure. The research on simple procedures to grow GaAs NWs with $d_{\mathrm{NW}}$ values below $100 \mathrm{~nm}$ and $L_{\mathrm{NW}}$ values above tenths of $\mu \mathrm{m}$, while preserving their crystallinity and electronic/optoelectronic properties, 
will allow for the development of high-performance electronic devices based on GaAs NWs, either directly grown on Si substrates or transferred to foreign substrates.

The growth of GaAs NWs through a bottom-up approach has been demonstrated by the wellknown metal-assisted vapour-liquid-solid (VLS) mechanism, using a metallic particle to assist the growth of the NW along a preferential direction determined by the orientation of the crystalline substrate. ${ }^{13}$ Vertically aligned GaAs NWs have been successfully grown on both $\mathrm{Si}(111)$ and GaAs(111)B substrates by different techniques, including molecular beam epitaxy (MBE) ${ }^{14-21}$ and chemical beam epitaxy (CBE) ${ }^{22-25}$ typically using $\mathrm{Au}$ and $\mathrm{Ga}$ as seed particles to form a supersaturated liquid alloy with growth species. Au has been extensively utilized in GaAs NW growths, but it could be incorporated as impurity into the NW structure,${ }^{26}$ and it could also induce zincblende/wurtzite (ZB/WZ) polytypisms along the NW body. These two important drawbacks support a wider use of Ga rather than Au for GaAs NW growth. Furthermore, theoretical models ${ }^{27}$ that were experimentally confirmed, ${ }^{24}$ have predicted and later demonstrated that Ga-assisted GaAs NWs present a better phase purity when Ga droplets partially wet the sidewalls of the NWs during the VLS based growth. However, the use of $\mathrm{Si}(111)$ with a thin capping oxide layer has shown the uncontrolled nucleation of other parasitic species, namely nanocrystals and traces, during Ga-assisted VLS growth of GaAs NWs by both $\mathrm{MBE}^{28}$ and $\mathrm{CBE}^{23-25}$ techniques, which could drastically affect some properties of resulting NWs. Pre-patterned $\mathrm{SiO}_{\mathrm{x}}$ thick layers on both $\mathrm{Si}(111)$ and $\mathrm{GaAs}(111) \mathrm{B}$ substrates have been extensively used to prevent the nucleation of parasitic species (Table 1).

The size uniformity obtained during Ga-assisted VLS growth of GaAs NWs has been recently analyzed as a function of precursor gas fluxes, in order to obtain highly uniform arrays of GaAs NWs on Si substrates. ${ }^{17}$ Further investigations about the role of the Ga catalyst contact angle on 
the resulting GaAs NW morphology have just demonstrated the possibility to grow high aspectratio GaAs NWs with $d_{\mathrm{NW}}$ below $20 \mathrm{~nm}$, allowing the observation of quantum confinement effects. ${ }^{15}$ All aforementioned achievements towards the accurate and reproducible growth of high quality GaAs NWs on Si substrates have been possible mainly due to the suitability of epitaxial techniques, including MBE and CBE, and to the study of different substrate pre-treatments. For the sake of comparison, Table 1 summarizes the performance obtained by different epitaxial techniques during the growth of vertically aligned GaAs NWs on $\mathrm{Si}(111)$ substrates by Ga-assisted VLS approach. This table also shows the pre-treatment of Si substrates prior to the growth to prevent the aforementioned parasitic species.

Table 1. Performance obtained from Ga-assisted VLS growths of GaAs NWs vertically aligned on either Si(111) or GaAs(111)B substrates, considering those approaches that successfully prevented the nucleation of parasitic species. The rows have been ordered as a function of the NW aspect ratio.

\begin{tabular}{|c|c|c|c|c|c|}
\hline Technique & Substrate pre-treatment & $\mathrm{NWs} / \mu \mathrm{m}^{2}$ & $\begin{array}{l}\text { NW radius } \\
(\mathrm{nm})\end{array}$ & $\begin{array}{l}\text { NW aspect } \\
\text { ratio }\end{array}$ & REF \\
\hline $\mathrm{MBE}$ & $\begin{array}{l}\text { e-beam lithography of nano-holes on } 10-\mathrm{nm} \\
\text { thick } \mathrm{SiO}_{x} / \mathrm{Si}(111)\end{array}$ & 3 & 10 & 100 & 15 \\
\hline $\mathrm{CBE}$ & 4-nm thick $\mathrm{SiO}_{\mathrm{x}} / \mathrm{Si}(111)$ & 0.4 & 35 & 85 & $\begin{array}{l}\text { This } \\
\text { Work }\end{array}$ \\
\hline MBE & $\begin{array}{l}\text { e-beam lithography of nano-holes on } 15-\mathrm{nm} \\
\text { thick } \mathrm{SiO}_{\mathrm{x}} / \mathrm{GaAs}(111) \mathrm{B}\end{array}$ & 9 & 40 & 63 & 18 \\
\hline MBE & $\begin{array}{l}\text { Nanoimprint lithography on } 40-n m \text { thick } \\
\qquad \mathrm{SiO}_{\mathrm{x}} / \mathrm{Si}(111)\end{array}$ & 3 & 70 & 39 & 16 \\
\hline MBE & $\begin{array}{l}\text { e-beam lithography of nano-holes on } 10-\mathrm{nm} \\
\text { thick } \mathrm{SiO}_{x} / \mathrm{Si}(111)\end{array}$ & 8 & 80 & 33 & 17 \\
\hline MBE & $\begin{array}{l}\text { e-beam lithography of nano-holes on 31-nm } \\
\text { thick } \mathrm{SiO}_{x} / \mathrm{Si}(111)\end{array}$ & 16 & 25 & 20 & 21 \\
\hline MBE & $\begin{array}{l}\text { e-beam lithography of nano-holes on 31-nm } \\
\text { thick } \mathrm{SiO}_{x} / \mathrm{Si}(111)\end{array}$ & 15 & 30 & 17 & 14 \\
\hline
\end{tabular}

As observed in Table 1, although the growth mechanisms of Ga-assisted GaAs NWs on Si(111) have been extensively analyzed as a function of growth conditions, only few studies have highlighted the nucleation of parasitic species and their role on the NW growth. Among all, advanced tools such as nano-lithography and high resolution photo-lithography have been successfully utilized to pattern nano-/micro-holes over a thick $\mathrm{SiO}_{\mathrm{x}}$ surface layer on $\mathrm{Si}(111)$ 
substrates, allowing the Ga-assisted VLS growth of GaAs NWs -mainly in MBE systems- to prevent parasitic species growth. ${ }^{14,16}$ Additionally, those approaches permit a precise positioning of NWs along the substrate surface, controlling therefore the resulting NW density, which is critical for the development of electronic devices. However, lithographic procedures are not suitable for growth techniques using uncracked metalorganic precursors, such as CBE, since the growth on pre-patterned $\mathrm{Si}$ substrates requires a $\mathrm{SiO}_{\mathrm{x}}$ layer with a thickness typically in the range of 20-30 nm, downgrading the cracking efficiency of metalorganic precursors on the substrate surface. The pre-patterning of nano-holes on $\mathrm{SiO}_{\mathrm{x}}$ has been rarely used to grow a high density of uniform GaAs NWs over large areas ( 2 inch wafer),${ }^{16}$ which is a feature required for applications such as large area photovoltaic cells. In this regard, the high-cost and incompatibility of techniques such as e-beam lithography to produce a massive number of nano-holes on $\mathrm{SiO}_{\mathrm{x}}$ thick layers (i.e. nanometric hole-to-hole pitch), limit their applicability to grow well-ordered arrays of vertically aligned GaAs NWs over large areas.

In a previous work, ${ }^{23}$ we have presented a thorough study on the growth mechanisms of GaAs NWs by Ga-assisted $\mathrm{CBE}$ on $\mathrm{Si}(111)$ substrates using triethylgallium (TEGa) and tertiarybuthylarsine (TBAs) as precursors, revealing the importance of the Si substrate surface preparation for the successful growth of NWs. In this work, we present an extended study on the NWs growth mechanism, introducing a novel method to preserve the growth conditions during long lasting VLS growths by hindering the formation of parasitic species, using neither nanolithography techniques nor complex substrate pre-treatments. Obtained NWs were analysed as a function of the growth conditions using scanning electron microscopy (SEM) to examine their morphology and density, and reflection high-energy electron diffraction (RHEED) to study their crystalline structure during the growth. 
The results shown in this work are an important step forward to the optimization of the resulting quality of GaAs NWs grown on Si substrates, preventing the use of expensive tools and complex engineering procedures, and contributing to the development of higher performance electronic devices.

\section{RESULTS AND DISCUSSION}

\subsection{One-step Growth Method}

One- and two-steps based growth procedures developed in this work are schematically described in FIG 1, after an initial Si oxide layer was previously grown on top of the Si substrate. The one-step growth, having been thoroughly described elsewhere ${ }^{23}$ consists in: i) substrate thermal annealing for 5 min at a substrate temperature $\left(T_{\mathrm{s}}\right)$ of $650^{\circ} \mathrm{C}$, in order to desorb remnant adsorbed molecules at the substrate surface (FIG 1(a)); ii) pre-deposition of TEGa equivalent to 7.5 Ga monolayers (MLs) at $T_{\mathrm{s}}=580{ }^{\circ} \mathrm{C}$, allowing for Ga droplets formation on the oxidized $\mathrm{Si}(111)$ surface, followed by a stabilization stage for a time $t_{\mathrm{s}}=90 \mathrm{~s}$ after TEGa valve is closed, leading to the rearrangement of Ga droplets on the substrate surface, and improving the resulting droplet size uniformity (FIG 1(b)); iii) finally, both TEGa and TBAs valves are opened at a V/III flux ratio of 0.8 , starting the VLS growth of NWs at a growth rate $\left(r_{\mathrm{g}}\right)$ of around $5.4 \mu \mathrm{m} / \mathrm{h}$ (FIG 1(c)). The value of $r_{\mathrm{g}}$ has been estimated from the final nanowire length $\left(L_{\mathrm{NW}}\right)$ determined by SEM images after the growth time. NW growth was carried out under a substrate rotation of around 5 rpm to ensure a better gas flux uniformity on the substrate surface. 
(a) Substrate Annealing

@ $T_{\mathrm{s}}=650^{\circ} \mathrm{C}$ for $5 \mathrm{~min}$

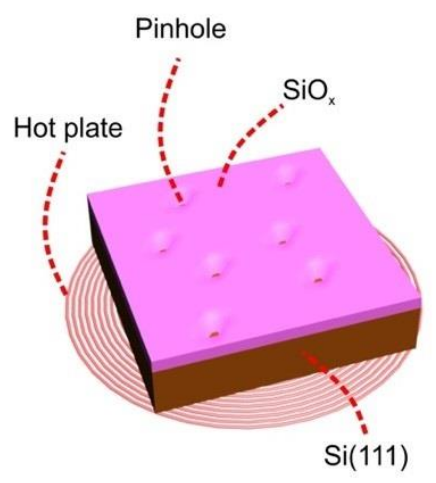

(d) Air Ambient Exposure

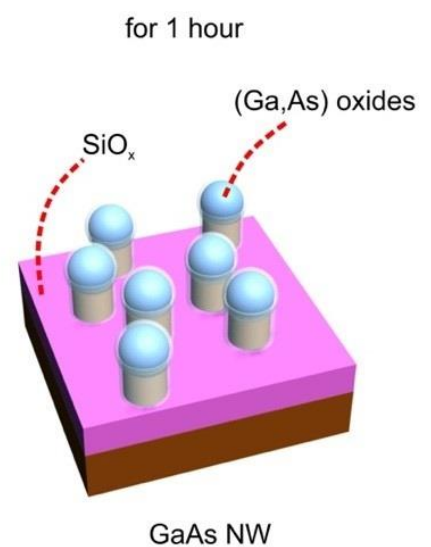

(b) Pre-deposition and

Stabilization

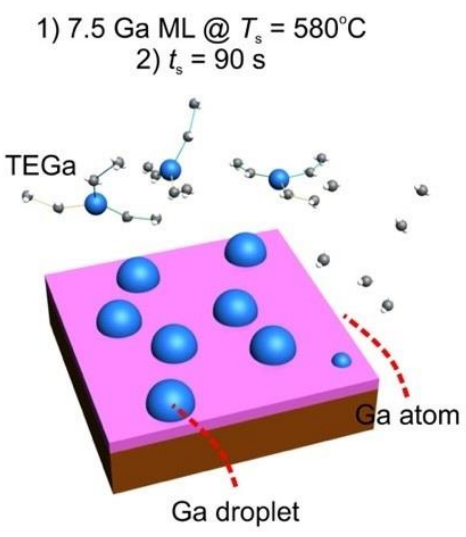

(e) Soft-annealing

@ $T_{\mathrm{s}}=600^{\circ} \mathrm{C}$ for $5 \mathrm{~min}$

(high vacuum conditions)

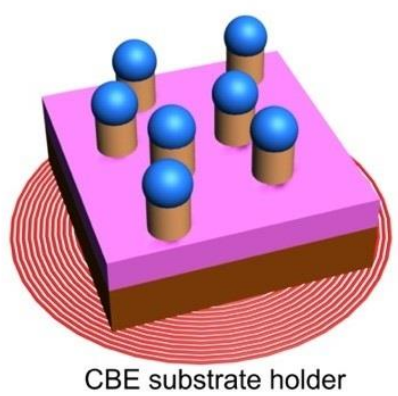

(c) GaAs NW Pre-growth

@ $\begin{gathered}T_{\mathrm{s}}=580^{\circ} \mathrm{C}, \mathrm{V} / \mathrm{III}=0.8 \\ r_{\mathrm{g}}=5.4 \mu \mathrm{m} / \mathrm{h}\end{gathered}$

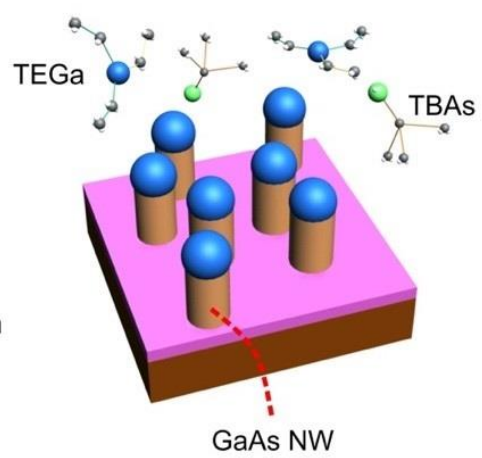

(f) GaAs NW re-growth

@ $T_{\mathrm{s}}=580^{\circ} \mathrm{C}, \mathrm{V} / \mathrm{III}=0.8$

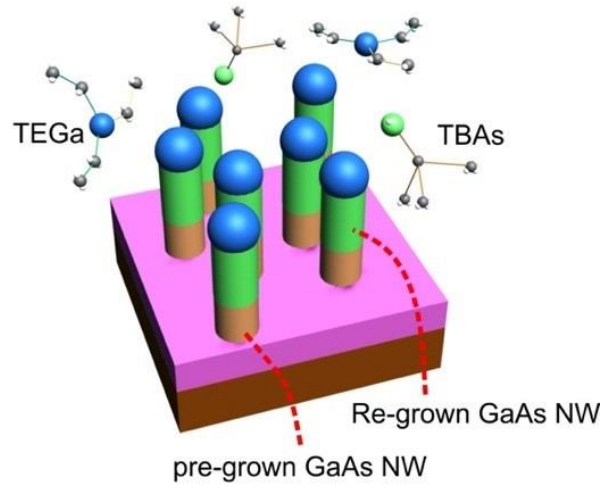

FIG 1. 3D Schematic illustration of the Ga-assisted CBE growth of GaAs NWs consisting in either (a-c) one-step or (a-f) two-steps based approaches. One-step approach consists of (a) $\operatorname{Si}(111)$ annealing at $T_{\mathrm{s}}=650{ }^{\circ} \mathrm{C}$ to create pinholes along the previously grown $\mathrm{SiO}_{\mathrm{x}}$ thin surface layer; (b) TEGa pre-deposition at $T_{\mathrm{s}}=580{ }^{\circ} \mathrm{C}$, resulting in $\mathrm{Ga}$ droplets randomly distributed along the substrate surface; (c) GaAs NW growth under TEGa and TBAs fluxes at $T_{\mathrm{s}}=580{ }^{\circ} \mathrm{C}$. Two-steps approach comprises (d) air exposure of the sample grown in (a-c) to increase the $\mathrm{SiO}_{\mathrm{x}}$ thickness; (e) soft-annealing at $T_{\mathrm{s}}=600{ }^{\circ} \mathrm{C}$ to desorb the oxide covering NWs and the Ga-catalyst; (f) regrowth of GaAs NWs using same conditions described in (c). 


\subsection{Two-steps Growth Method}

The two-steps growth method starts with a GaAs NWs pre-growth carried out during 4 min under the same conditions than those previously described for the one-step growth method (FIG 1(a-c)). After this first step, the sample temperature is decreased to room temperature at the fastest allowed rate to reduce any residual growth as much as possible. After cooling down, the sample is taken out of the CBE system to be exposed to ambient air conditions for 1 hour to promote a controlled re-oxidation. This extra oxidation step increases the $\mathrm{SiO}_{\mathrm{x}}$ thickness at those areas where NWs did not grow (FIG 1(d)). GaAs NWs and Ga droplets surfaces are also expected to be

oxidized during this process. Thereafter, the samples are transferred again to the CBE system and annealed under ultra high vacuum for $5 \mathrm{~min}$ at $T_{\mathrm{s}}=600^{\circ} \mathrm{C}$. It is well known that native or chemical Ga and As oxide containing layers covering GaAs are usually desorbed at $580^{\circ} \mathrm{C}$ prior to epitaxial growth, while Si oxide layers need much higher temperatures, in the $750-800{ }^{\circ} \mathrm{C}$ range. The annealing temperature used here, $T_{\mathrm{s}}=600^{\circ} \mathrm{C}$, is expected to be high enough for desorption of $\mathrm{Ga}$ and As oxides covering NW sidewalls and Ga droplet, while it preserves Si oxide on the Si substrate surface (FIG 1(e)). It is worth noting that Si oxide thickness at this point is expected to be larger than the initial thickness before Ga droplet formation. Finally, $T_{\mathrm{s}}$ is lowered down to 580 ${ }^{\circ} \mathrm{C}$ and a "re-growth" is carried out for different times by opening both TEGa and TBAs valves, using the same V/III flux ratio of 0.8 (FIG 1(f)).

\subsection{Ga Droplet Nucleation on Si(111)}

In a Ga-assisted growth, the formation of Ga droplets on top of the Si substrate surface is necessary to allow for the VLS growth of GaAs NWs. ${ }^{23-25}$ Specially for CBE growth, the preparation of the $\mathrm{Si}(111)$ substrate prior to the growth process -i.e. the preparation of a thin film 
of $\mathrm{SiO}_{\mathrm{x}}$ - is crucial to enable the thermal decomposition of TEGa molecules on top of the oxidized Si surface, resulting in Ga droplets randomly distributed along the substrate surface (FIG 1(b)). ${ }^{29}$ The chemical composition of the resultant $\operatorname{Si}(111)$ surface oxide after the application of etching protocols described above was analyzed by FTIR (see FIG S1(a)) and ATR (see FIG S1(b)). FTIR measurements show a diffuse reflectance signal with two main peaks centred around 1100 and $1250 \mathrm{~cm}^{-1}$ which are related to interstitial $\mathrm{O}(\mathrm{Si}-\mathrm{O}-\mathrm{Si})$ and stoichiometric $\mathrm{SiO}_{2}$, respectively. ${ }^{30}$ These results are in good agreement with previous works, where similar chemical treatments result in a $\mathrm{SiO}_{\mathrm{x}}$ thin film with a random distribution of pinholes where Ga droplets can be preferentially

formed. ${ }^{29}$ The nucleation of Ga droplets on top of $\mathrm{Si}(111)$ substrates has been confirmed in-situ by RHEED and $e x$-situ by SEM elsewhere. ${ }^{23}$ In those previous studies, we also analyzed Ga droplets size and their surface density as a function of Ga pre-deposition time and stabilization time, because they are crucial for controlling the distribution of NWs and their diameter.

\subsection{One-step Growth Performance}

During the NW growth, other GaAs features such as traces and nanocrystals (called here parasitic species) can also nucleate in the NWs vicinity (FIG 2(a,b)). ${ }^{24,25}$ All tilted SEM images shown in this work, as FIG 2(b), were taken $30^{\circ}$ away from the substrate surface normal direction. As this tilting value reduces the apparent vertical dimensions by a factor of $\sin 30^{\circ}=0.5$, the real vertical scale has been multiplied by 2 in all tilted SEM images.

The nucleation of parasitic species was also observed in MBE processes using both $\operatorname{thin}^{28}$ or thick dielectric layers, ${ }^{29}$ and it has been linked to the chemical interaction between the Ga droplets and the dielectric layer. From FIG 2(a) one can conclude that there are two different collections of nanocrystals rotated $60^{\circ}$ between each other, whose surfaces develop edges that are aligned with the hexagonal footprint of the NWs, indicating that nanocrystals have also nucleated on the $\mathrm{Si}$ 
substrate in regions with a thinner or etched oxide. The density of parasitic species tends to increase over the growth time, which could be an important issue to preserve the initial growth conditions, thus limiting the maximum length of the NWs and hindering their aspect ratio. In this regard, we have previously reported ${ }^{23}$ that GaAs NWs grown through a one-step method show an initially high $r_{\mathrm{g}}$, while for growth times above $15 \mathrm{~min}$ a progressive reduction of $r_{\mathrm{g}}$ is observed, related to the nucleation of nanocrystalline GaAs. Nucleated GaAs nanocrystals increase their number and size as growth proceeds, until a layer covering the whole substrate surface is formed (see FIG S3), limiting the maximum achievable NW length to approximately $3 \mu \mathrm{m}$.

The formation of parasitic species was also confirmed by RHEED after 5-6 min of growth, when new spots in the diffraction pattern are observed, such as titled lines underneath the $\alpha$ and $\beta$ lines and inter-spots labelled as $\delta$ in FIG 2(c). As the growth of parasitic species also consumes both $\mathrm{Ga}$ and As atoms, a reduction on the incorporation of these species to the droplet/NW interface will take place, altering the effective V/III ratio and decreasing the Ga droplet size. Since the NW diameter is affected by the size of the Ga droplet, its decrease also implies a reduction on the NW diameter, resulting in the tapering effect clearly observed in FIG 2(b). Furthermore, if the effective incorporation of $\mathrm{Ga}$ to the droplet is not sufficient, the droplet can be completely consumed by the VLS growth, finishing the longitudinal growth of the NWs when their length reaches about $3 \mu \mathrm{m}$. In addition to the above effect, the axial growth of the NW can be especially promoted at those regions near the NW base, which can be an additional factor contributing to the tapering effect observed in FIG 2(b). Another possible explanation for NWs tapering could be related to the change on the effective V/III ratio during NWs growth, due to the competition with parasitic species to incorporate Ga and As atoms. 

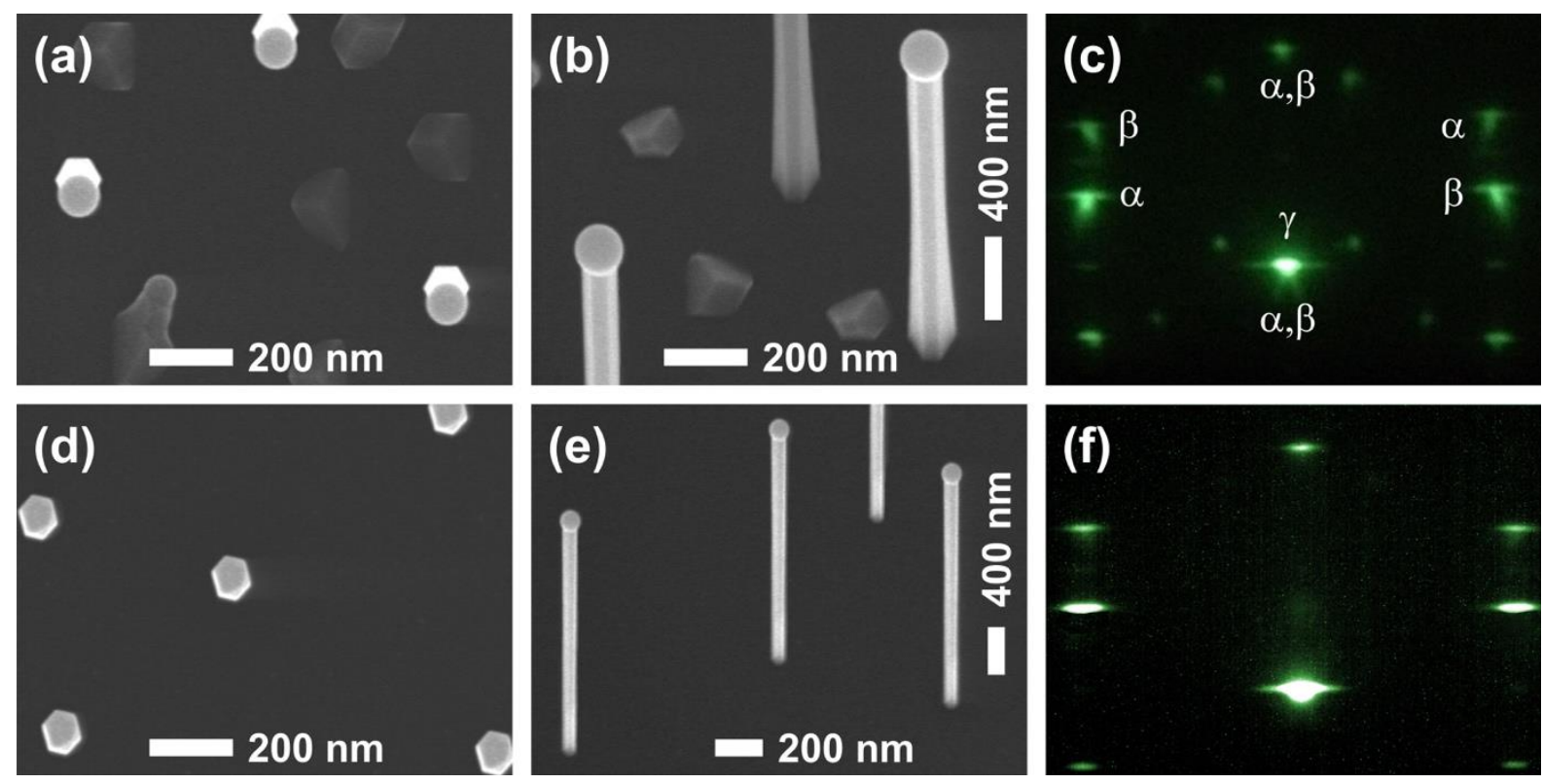

FIG 2. SEM images of GaAs NWs grown by $(a, b)$ one-step and (d,e) two-steps growth methods taken at $(\mathrm{a}, \mathrm{d}) 0^{\circ}$, and $(\mathrm{b}, \mathrm{e}) 30^{\circ}$ with respect to the substrate normal direction; (c) and (f) RHEED patterns corresponding to one-step and two-steps growth methods, respectively.

\subsection{Two-steps Growth Performance}

In the two-steps growth method, the scenario is very different from the one-step growth. The reduced 4 min growth time for the first growth step allows for the growth of short NWs (FIG 1(c)) while reducing the formation of parasitic species, whose growth rates are much slower, and their nucleation may be delayed. After the sample is exposed to the ambient air for 1 hour to increase the thickness of the $\mathrm{SiO}_{\mathrm{x}}$ layer (FIG 1(d)), the small and thin parasitic structures, if any, that could have nucleated previously during the first growth step may also be fully oxidized, and allowed to be desorbed during the next annealing step.

After Ga and As oxide desorption, the re-growth step was then carried out using the same conditions than those used during pre-growth. FIG 2(d,e) show SEM images of resulting NWs, 
observing a higher aspect ratio $\left(L_{\mathrm{NW}} / d_{\mathrm{NW}}=85\right.$, for 56-min re-growth time) and growth rates $\left(r_{g} \sim\right.$ $6 \mu \mathrm{m} / \mathrm{h})$ than those obtained under the one-step growth method $\left(L_{\mathrm{NW}} / d_{\mathrm{NW}}=15\right.$ and $r_{g}=3 \mu \mathrm{m} / \mathrm{h}$, respectively). This result can be explained on the basis of a drastic reduction of parasitic species under the two-steps growth method, which preserves the VLS conditions at the catalyst, allowing the growth of NWs with a better aspect ratio at a higher and constant growth rate, while observing no tapering effect (FIG $2(\mathrm{~d}, \mathrm{e}))$.

From FIG 2(d,e) one can also conclude that the nucleation of parasitic species was totally inhibited by the two-steps growth procedure. FIG 2(f) presents the characteristic RHEED diffraction pattern under the $\langle 1-10\rangle$ azimuth observed during the whole growth under the twosteps method; as mentioned before, horizontal lines correspond to NWs, whereas there was no evidence of inter-spots neither lines underneath the ZB lines, which confirms the absence of parasitic species. From above observations, RHEED has proved to be a precise tool to survey the formation of different species during NWs growth. 
(a) Two-steps growth

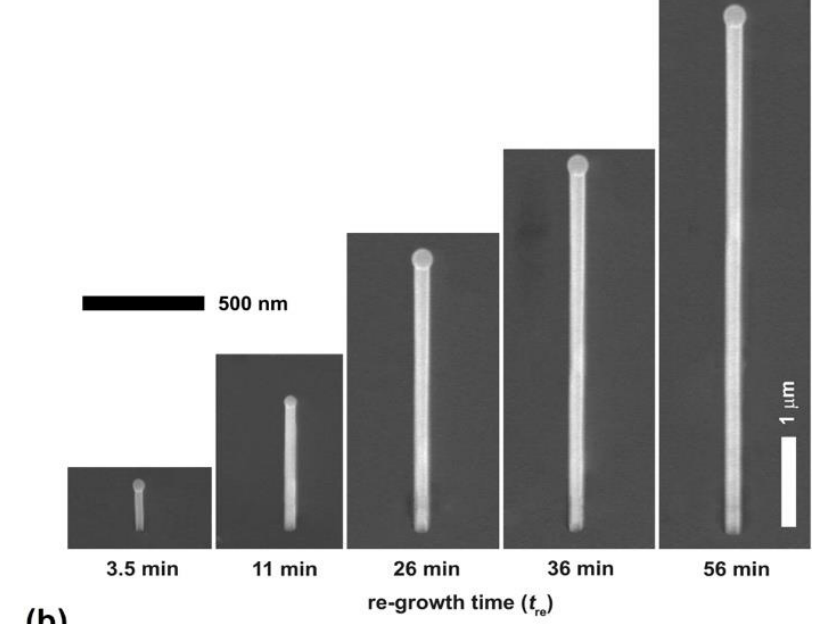

(b)

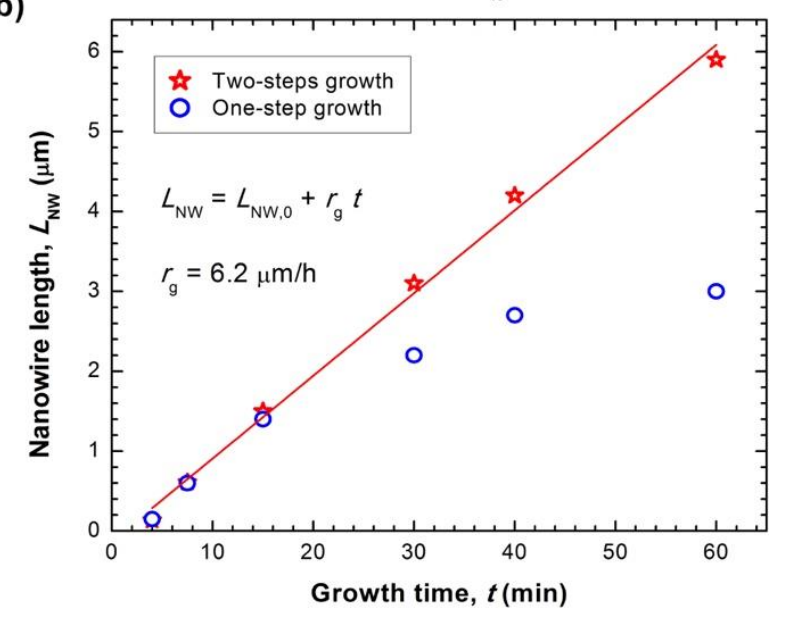

FIG 3. (a) SEM images of GaAs NWs grown under two-steps growth for different growth times;

(b) NW length vs total growth time for GaAs NWs grown by one-step (blue circles) and twosteps (red starts) growth methods.

GaAs NWs were grown for different re-growth times $\left(t_{\mathrm{re}}\right)$ ranged between $3.5,11,26$, 36, and 56 min after a pre-growth time $\left(t_{\text {pre }}\right)$ of 4 min, resulting in a total growth time $\left(t=t_{\text {pre }}+t_{\text {re }}\right)$ of 7.5 , 15, 30, 40, and 60 min, respectively. Resulting NWs are shown in FIG 3(a), each NW image represents the average NW found for every sample, being representative of the observed NW population. FIG 3(b) represents the dependence of $L_{\mathrm{NW}}$ obtained from SEM images of GaAs NWs 
grown under one-step (blue circles) and two-steps (red starts) growths, as a function of the growth time. In the two-steps growth procedure, $L_{\mathrm{NW}}$ exhibits a linear trend with the growth time, where $r_{\mathrm{g}}$ is around $6.2 \mu \mathrm{m} / \mathrm{h}$. While both one-step and two-steps growth methods present approximately the same initial $r_{\mathrm{g}}$ value for growth times below $30 \mathrm{~min}$, only the latter can preserve this stable $r_{\mathrm{g}}$ value, whereas $r_{\mathrm{g}}$ tends to decrease in the former, until the NW growth is stopped for growth times above $60 \mathrm{~min}$.

The observed linear growth kinetics for NWs has also been reported by previous experimental works, ${ }^{31-33}$ and it has also been predicted by using theoretical models. ${ }^{34}$

The promising result obtained through the two-steps growth method is accompanied by three more characteristics, namely the achievement of lower NW diameters, the observation of a drastic decrease -or even suppression- of parasitic species surrounding the NWs, and a high uniformity in density, size and height of NWs. These observations are clearly seen in FIG 4, which shows a wide field image of a sample with NWs grown during 15 min by the two-steps growth method, with an average density of $0.4 \mathrm{NW} / \mu \mathrm{m}^{2}$.

Two-steps grown NWs described in this work have been successfully used to build a NWbased photodetector ${ }^{35}$ by using NWs ultrasound lift-off in ethanol, followed by dielectrophoretic assembly of NWs on previously patterned contacts.

Summarizing, the best outcome obtained in our CBE system using the novel proposed twosteps growth method -included in Table 1- has shown a high density array of uniform and crystalline GaAs NWs vertically aligned on large area $\operatorname{Si}(111)$ substrates $\left(\sim 1 \mathrm{~cm}^{2}\right)$. SEM characterization of resulting samples shows an average $\mathrm{NW}$ density $0.4 \mathrm{NW} / \mu \mathrm{m}^{2}$, with a homogeneous NW radius of $35 \mathrm{~nm}$, and a uniform aspect ratio of 85 , which are close to record values obtained using MBE. 


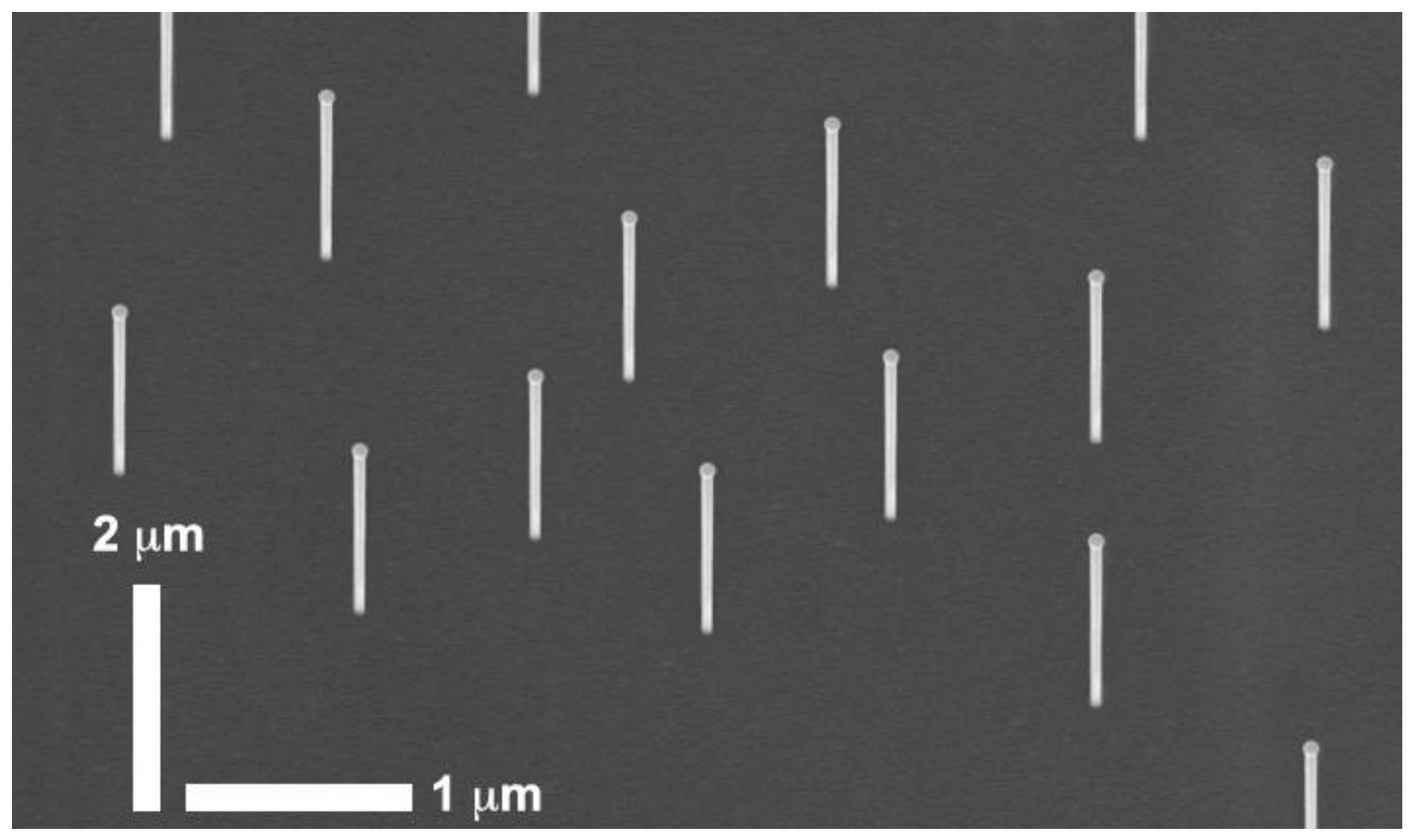

FIG 4. SEM image of GaAs NWs grown for $15 \mathrm{~min}$ on $\mathrm{Si}(111)$ by the described two-steps method. 


\section{CONCLUSIONS}

We have developed a novel method to grow GaAs NWs by Ga-assisted CBE improving both $\mathrm{NW}$ aspect ratio (up to 85$)$ and constant growth rate $(\sim 6.2 \mu \mathrm{m} / \mathrm{h})$, obtaining NWs with lengths up to $6.2 \mu \mathrm{m}$ and radius of $35 \mathrm{~nm}$ after a 1 hour growth. The proposed growth method is based on the growth of a thin $\mathrm{SiO}_{\mathrm{x}}$ layer after a short pre-growth of GaAs NWs, and before a second and longer GaAs NW re-growth. This procedure has been demonstrated to reduce drastically the nucleation of parasitic GaAs nano-/micro-structures on the $\mathrm{Si}(111)$ substrate surface -as demonstrated in-situ by RHEED and ex-situ by SEM- and without the utilization of any complex and expensive tool such as nano-lithography. The simple approach proposed in this work favours the continuous and effective supply of Ga and As atoms to the growth interface formed between the NW and the Gacatalyst, preserving VLS conditions and a nearly constant growth rate. As these are critical conditions required in for Ga-assisted VLS growths carried out in MBE and rarely achieved in $\mathrm{CBE}$, our approach is promising for obtaining high-aspect ratio and high quality GaAs NWs on Si substrates.

\section{MATERIALS AND METHODS}

\subsection{Growth system}

GaAs NWs have been grown in a RIBER CBE32 system by Ga-assisted CBE on oxidized $\mathrm{Si}(111)$ substrates, using triethylgallium (TEGa) and tertiarybuthylarsine (TBAs) as Ga and As metalorganic precursors, respectively. TEGa and TBAs fluxes are controlled by a pressure regulated control system using an intermediate chamber for each precursor, located between each liquid container and the growth chamber and a calibrated hole. TBAs flux is directed to the substrate surface using a high temperature gas cracking cell $\left(T_{\mathrm{HT}}=820^{\circ} \mathrm{C}\right)$ to obtain reactive As- 
containing species by pyrolysis, while uncracked TEGa flux is injected through a low temperature gas cell $\left(T_{\mathrm{LT}}=80{ }^{\circ} \mathrm{C}\right)$ to be dissociated on the substrate surface at the growth temperature $T_{\mathrm{s}}$, ranged between $580{ }^{\circ} \mathrm{C}<T_{\mathrm{s}}<620^{\circ} \mathrm{C}$, which is measured by using a dual wavelength pyrometer.

\subsection{Substrate Preparation}

Samples used in this work consist in triangular-shaped cleaved pieces of $\mathrm{Si}(111)$ wafers (from Virginia Semiconductor), whose sides are limited by $\{-211\}$ planes; the use of this kind of substrates allows for an easy identification of GaAs NW crystalline planes, relative to the Si substrate.

Prior to growth, substrates are etched in a $10 \% \mathrm{HF}$ aqueous solution for 5 min and $\mathrm{N}_{2}$ blow dried to remove the native oxide layer $\left(\mathrm{SiO}_{\mathrm{x}}\right)$ capping the $\mathrm{Si}$ surface. The substrate surface after the above chemical etch is analyzed by means of Fourier transform infrared spectroscopy (FTIR), attenuated total reflectance (ATR) (see FIG S1(a,b)) and spectroscopic ellipsometry (SE) (see FIG $\mathrm{S} 2$ ). FTIR and ATR techniques are employed to study the chemical structure of $\mathrm{SiO}_{\mathrm{x}}$ after the etching procedure, while SE is used to determine both residual oxide thickness and roughness. After etching, each substrate is In-bonded onto a molybdenum sample holder on a hot plate at 200 ${ }^{\circ} \mathrm{C}$. Once the substrate is cooled down to room temperature, it is etched again for 5 min by using the above HF solution, in order to remove the oxide grown during the bonding stage. Prior to introduction in the $\mathrm{CBE}$ system under high vacuum conditions, substrates are exposed to the ambient air conditions for $5 \mathrm{~min}$ to obtain the final $\mathrm{SiO}_{\mathrm{x}}$ capping layer with a thickness of $1.5 \mathrm{~nm}$, as determined by SE (see FIGS2), in a controlled way. 


\section{Supporting Information}

(a)

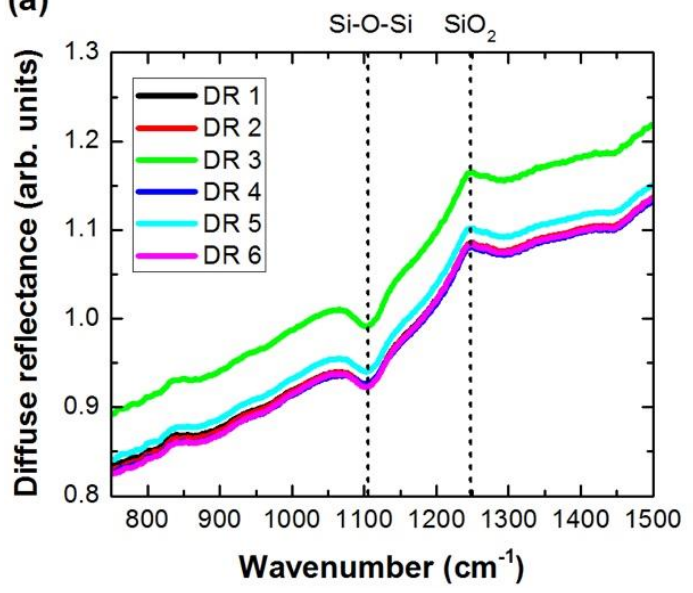

(b)

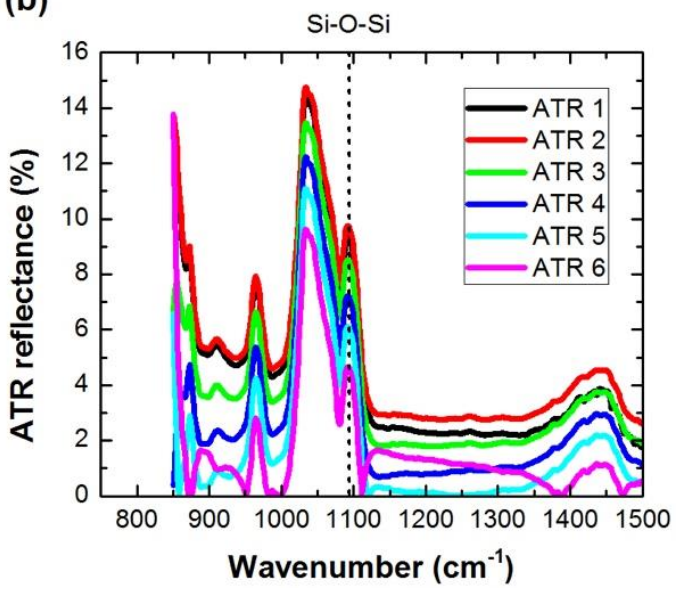

FIG S1. Fourier-transform infrared spectroscopy spectra of $\mathrm{SiO}_{\mathrm{x}}$ for determination of oxide thickness on Si wafers.

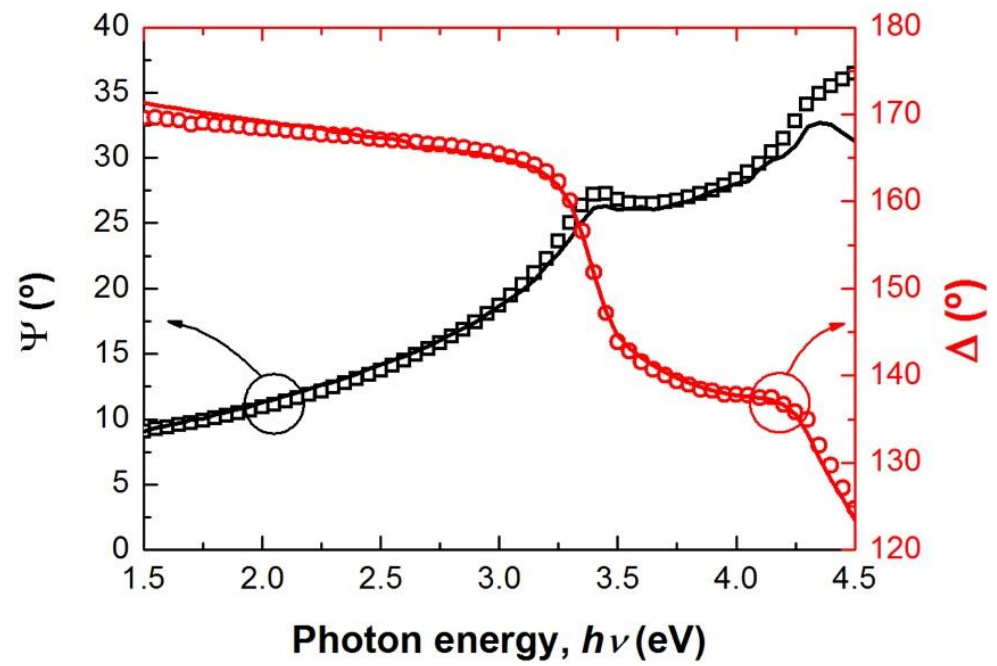

FIG S2. Spectroscopic ellipsometry measurements on a $\mathrm{SiO}_{\mathrm{x}} / \mathrm{Si}(111)$ sample after etching procedure (squares), and fitting a two-layer model (lines). 


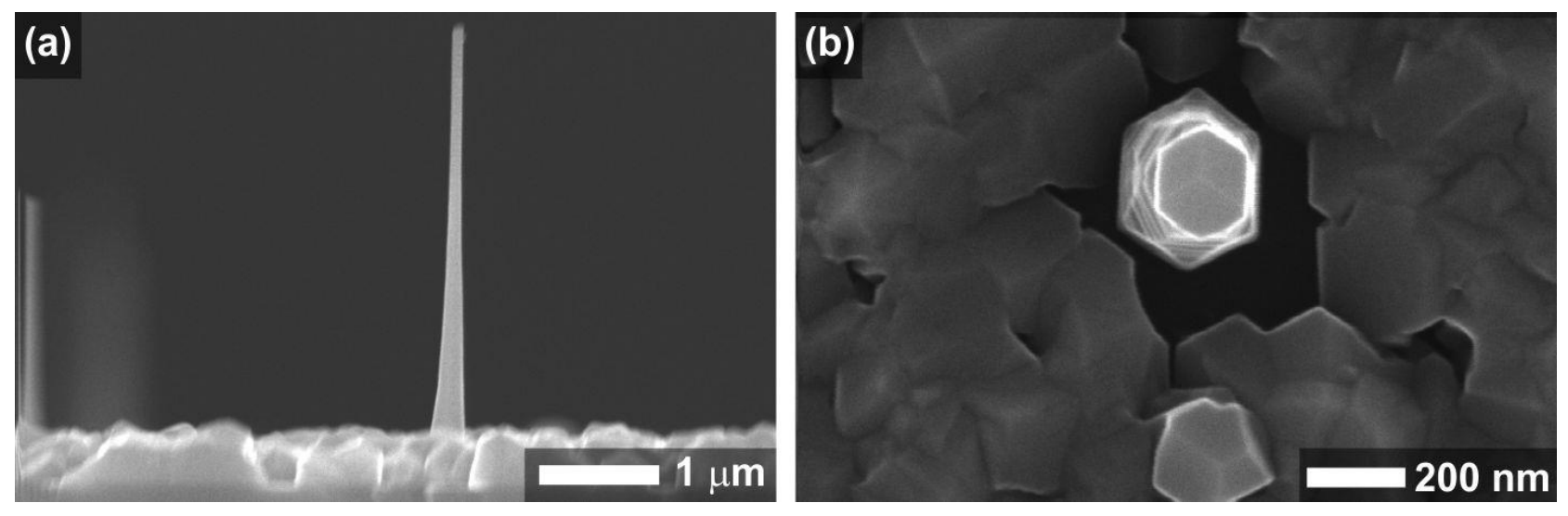

FIG S3. (a) cross-section and (b) top-view SEM images of a single GaAs NW grown under onestep growth conditions above $60 \mathrm{~min}$. 


\section{AUTHOR INFORMATION}

\section{*Corresponding Author}

Prof. Basilio Javier García

Department of Applied Physics, Faculty of Sciences

Universidad Autónoma de Madrid

C/ Francisco Tomás y Valiente, 7, 28049 Madrid, Spain

e-mail: basilio.javier.garcia@uam.es

\section{Present Addresses}

${ }^{\dagger}$ Micromanipulation Group, Electronics and Nanoscale Engineering, University of Glasgow, G128QQ Glasgow, United Kingdom.

\section{Author Contributions}

C.G.N. and B.J.G conceptualized the work; C.G.N. and B.J.G. carried out the synthesis and characterization of nanowires with support from A.F.B and N.L.; C.G.N. and B.J.G. wrote the manuscript with support from all co-authors; B.J.G. provided overall supervision for the project; All authors have given approval to the final version of the manuscript.

\section{Funding Sources}

Research work supported by the Spanish Ministerio de Ciencia e Innovación (TEC2010-20796).

\section{Acknowledgment}

This research was supported by the TEC2010-20796 (FPI grant), funded by the Spanish Ministerio de Ciencia e Innovación (MICINN). Authors are also thankful to the Servicio 
Interdepartamental de Investigación (SIdI) at Universidad Autónoma de Madrid for technical support on the characterization of nanowires. 


\section{References}

1. Lu, W.; Lieber, C. M., Semiconductor nanowires. Journal of Physics D: Applied Physics 2006, 39 (21), R387.

2. Zhang, A.; Lieber, C. M., Nano-bioelectronics. Chemical reviews 2015, 116 (1), 215-257.

3. Li, Y.; Qian, F.; Xiang, J.; Lieber, C. M., Nanowire electronic and optoelectronic devices. Materials today 2006, 9 (10), 18-27.

4. Tian, B.; Kempa, T. J.; Lieber, C. M., Single nanowire photovoltaics. Chemical Society Reviews 2009, 38 (1), 16-24.

5. Heiss, M.; Fontana, Y.; Gustafsson, A.; Wüst, G.; Magen, C.; O’regan, D.; Luo, J.; Ketterer, B.; Conesa-Boj, S.; Kuhlmann, A., Self-assembled quantum dots in a nanowire system for quantum photonics. Nature materials 2013, 12 (5), 439.

6. García Núñez, C.; García Marín, A.; Nanterne, P.; Piqueras, J.; Kung, P.; Pau, J. L., Conducting Properties of Nearly Depleted ZnO Nanowire UV Sensors Fabricated by Dielectrophoresis. Nanotechnology 2013, 24 (41), 415702.

7. Krogstrup, P.; Jørgensen, H. I.; Heiss, M.; Demichel, O.; Holm, J. V.; Aagesen, M.; Nygard, J.; i Morral, A. F., Single-nanowire solar cells beyond the Shockley-Queisser limit. Nature Photonics 2013, 7 (4), 306-310.

8. Wang, H., High gain single GaAs nanowire photodetector. Applied Physics Letters 2013, $103(9), 093101$.

9. Björk, M.; Ohlsson, B.; Thelander, C.; Persson, A.; Deppert, K.; Wallenberg, L.; Samuelson, L., Nanowire resonant tunneling diodes. Applied Physics Letters 2002, 81 (23), 44584460 .

10. Kasai, S.; Hasegawa, H., A single electron binary-decision-diagram quantum logic circuit based on Schottky wrap gate control of a GaAs nanowire hexagon. Electron Device Letters, IEEE 2002, 23 (8), 446-448.

11. Heinrich, J.; Huggenberger, A.; Heindel, T.; Reitzenstein, S.; Höfling, S.; Worschech, L.; Forchel, A., Single photon emission from positioned GaAs/AlGaAs photonic nanowires. Applied Physics Letters 2010, 96 (21), 211117.

12. Tang, Y.; Chen, Z.; Song, H.; Lee, C.; Cong, H.; Cheng, H.; Zhang, W.; Bello, I.; Lee, S., Vertically aligned p-type single-crystalline $\mathrm{GaN}$ nanorod arrays on n-type $\mathrm{Si}$ for heterojunction photovoltaic cells. Nano letters 2008, 8 (12), 4191-4195.

13. Wagner, R. S.; Ellis, W. C., VAPOR-LIQUID-SOLID MECHANISM OF SINGLE CRYSTAL GROWTH. Applied Physics Letters 1964, 4 (5), 89-90.

14. Plissard, S.; Larrieu, G.; Wallart, X.; Caroff, P., High yield of self-catalyzed GaAs nanowire arrays grown on silicon via gallium droplet positioning. Nanotechnology 2011, 22 (27), 275602.

15. Kim, W.; Dubrovskii, V. G.; Vukajlovic-Plestina, J.; Tütüncüoglu, G.; Francaviglia, L.; Güniat, L.; Potts, H.; Friedl, M.; Leran, J.-B.; Fontcuberta i Morral, A., Bi-stability of contact angle and its role in achieving quantum-thin self-assisted GaAs nanowires. Nano letters 2018.

16. Munshi, A. M.; Dheeraj, D. L.; Fauske, V. T.; Kim, D.-C.; Huh, J.; Reinertsen, J. F.; Ahtapodov, L.; Lee, K.; Heidari, B.; Van Helvoort, A., Position-controlled uniform GaAs nanowires on silicon using nanoimprint lithography. Nano letters 2014, 14 (2), 960-966.

17. Vukajlovic-Plestina, J.; Kim, W.; Dubrovski, V. G.; Tütüncüoğlu, G.; Lagier, M.; Potts, H.; Friedl, M.; Fontcuberta i Morral, A., Engineering the Size Distributions of Ordered GaAs Nanowires on Silicon. Nano Letters 2017, 17 (7), 4101-4108. 
18. Bauer, B.; Rudolph, A.; Soda, M.; i Morral, A. F.; Zweck, J.; Schuh, D.; Reiger, E., Position controlled self-catalyzed growth of GaAs nanowires by molecular beam epitaxy. Nanotechnology 2010, 21 (43), 435601.

19. Russo-Averchi, E.; Vukajlovic Plestina, J.; Tütüncüoglu, G.; Matteini, F.; DalmauMallorquí, A.; de la Mata, M.; Rüffer, D.; Potts, H. A.; Arbiol, J.; Conesa-Boj, S.; Fontcuberta i Morral, A., High Yield of GaAs Nanowire Arrays on Si Mediated by the Pinning and Contact Angle of Ga. Nano Letters 2015, 15 (5), 2869-2874.

20. Gibson, S.; LaPierre, R., Study of radial growth in patterned self-catalyzed GaAs nanowire arrays by gas source molecular beam epitaxy. physica status solidi (RRL) - Rapid Research Letters 2013, 7 (10), 845-849.

21. Dubrovskii, V.; Xu, T.; Álvarez, A. D.; Plissard, S.; Caroff, P.; Glas, F.; Grandidier, B., Self-equilibration of the diameter of Ga-catalyzed GaAs nanowires. Nano letters 2015, 15 (8), 5580-5584.

22. Persson, A.; Ohlsson, B.; Jeppesen, S.; Samuelson, L., Growth mechanisms for GaAs nanowires grown in CBE. Journal of crystal growth 2004, 272 (1), 167-174.

23. García Núñez, C.; Braña, A. F.; López, N.; García, B. J., GaAs nanowires grown by Gaassisted chemical beam epitaxy: Substrate preparation and growth kinetics. J. Cryst. Growth 2015, 430, 108-115.

24. García Núñez, C.; Braña, A. F.; Pau, J. L.; Ghita, D.; García, B. J.; Shen, G.; Wilbert, D. S.; Kim, S. M.; Kung, P., Pure zincblende GaAs nanowires grown by Ga-assisted chemical beam epitaxy. Journal of Crystal Growth 2013, 372, 205-212.

25. García Núñez, C.; Braña, A. F.; Pau, J. L.; Ghita, D.; García, B. J.; Shen, G.; Wilbert, D. S.; Kim, S. M.; Kung, P., Surface optical phonons in GaAs nanowires grown by Ga-assisted chemical beam epitaxy. Journal of Applied Physics 2014, 115 (3), 034307.

26. Perea, D. E.; Allen, J. E.; May, S. J.; Wessels, B. W.; Seidman, D. N.; Lauhon, L. J., Threedimensional nanoscale composition mapping of semiconductor nanowires. Nano letters 2006, 6 (2), 181-185.

27. Dubrovskii, V.; Cirlin, G.; Sibirev, N.; Jabeen, F.; Harmand, J.; Werner, P., New mode of vapor- liquid- solid nanowire growth. Nano letters 2011, 11 (3), 1247-1253.

28. Matteini, F.; Dubrovskii, V. G.; Rüffer, D.; Tütüncüoğlu, G.; Fontana, Y.; Morral, A. F. I., Tailoring the diameter and density of self-catalyzed GaAs nanowires on silicon. Nanotechnology 2015, 26 (10), 105603.

29. Fontcuberta i Morral, A.; Colombo, C.; Abstreiter, G.; Arbiol, J.; Morante, J., Nucleation mechanism of gallium-assisted molecular beam epitaxy growth of gallium arsenide nanowires. Applied Physics Letters 2008, 92 (6), 063112-063112-3.

30. Matteini, F.; Tutuncuoglu, G.; Rüffer, D.; Alarcon-Llado, E.; Morral, A., Untangling the role of oxide in Ga-assisted growth of GaAs nanowires on Si substrates. arXiv preprint arXiv:1307.61132013.

1. Lu, W.; Lieber, C. M., Semiconductor nanowires. Journal of Physics D: Applied Physics 2006, 39 (21), R387.

2. Zhang, A.; Lieber, C. M., Nano-bioelectronics. Chemical reviews 2015, 116 (1), 215-257.

3. Li, Y.; Qian, F.; Xiang, J.; Lieber, C. M., Nanowire electronic and optoelectronic devices. Materials today 2006, 9 (10), 18-27.

4. Tian, B.; Kempa, T. J.; Lieber, C. M., Single nanowire photovoltaics. Chemical Society Reviews 2009, 38 (1), 16-24. 
5. Heiss, M.; Fontana, Y.; Gustafsson, A.; Wüst, G.; Magen, C.; O’regan, D.; Luo, J.; Ketterer, B.; Conesa-Boj, S.; Kuhlmann, A., Self-assembled quantum dots in a nanowire system for quantum photonics. Nature materials 2013, 12 (5), 439.

6. García Núñez, C.; García Marín, A.; Nanterne, P.; Piqueras, J.; Kung, P.; Pau, J. L., Conducting Properties of Nearly Depleted $\mathrm{ZnO}$ Nanowire UV Sensors Fabricated by Dielectrophoresis. Nanotechnology 2013, 24 (41), 415702.

7. Krogstrup, P.; Jørgensen, H. I.; Heiss, M.; Demichel, O.; Holm, J. V.; Aagesen, M.; Nygard, J.; i Morral, A. F., Single-nanowire solar cells beyond the Shockley-Queisser limit. Nature Photonics 2013, 7 (4), 306-310.

8. Wang, H., High gain single GaAs nanowire photodetector. Applied Physics Letters 2013, $103(9), 093101$.

9. Björk, M.; Ohlsson, B.; Thelander, C.; Persson, A.; Deppert, K.; Wallenberg, L.; Samuelson, L., Nanowire resonant tunneling diodes. Applied Physics Letters 2002, 81 (23), 44584460 .

10. Kasai, S.; Hasegawa, H., A single electron binary-decision-diagram quantum logic circuit based on Schottky wrap gate control of a GaAs nanowire hexagon. Electron Device Letters, IEEE 2002, 23 (8), 446-448.

11. Heinrich, J.; Huggenberger, A.; Heindel, T.; Reitzenstein, S.; Höfling, S.; Worschech, L.; Forchel, A., Single photon emission from positioned GaAs/AlGaAs photonic nanowires. Applied Physics Letters 2010, 96 (21), 211117.

12. Tang, Y.; Chen, Z.; Song, H.; Lee, C.; Cong, H.; Cheng, H.; Zhang, W.; Bello, I.; Lee, S., Vertically aligned p-type single-crystalline $\mathrm{GaN}$ nanorod arrays on n-type $\mathrm{Si}$ for heterojunction photovoltaic cells. Nano letters 2008, 8 (12), 4191-4195.

13. Wagner, R. S.; Ellis, W. C., VAPOR-LIQUID-SOLID MECHANISM OF SINGLE CRYSTAL GROWTH. Applied Physics Letters 1964, 4 (5), 89-90.

14. Plissard, S.; Larrieu, G.; Wallart, X.; Caroff, P., High yield of self-catalyzed GaAs nanowire arrays grown on silicon via gallium droplet positioning. Nanotechnology 2011, 22 (27), 275602.

15. Kim, W.; Dubrovskii, V. G.; Vukajlovic-Plestina, J.; Tütüncüoglu, G.; Francaviglia, L.; Güniat, L.; Potts, H.; Friedl, M.; Leran, J.-B.; Fontcuberta i Morral, A., Bi-stability of contact angle and its role in achieving quantum-thin self-assisted GaAs nanowires. Nano letters 2018.

16. Munshi, A. M.; Dheeraj, D. L.; Fauske, V. T.; Kim, D.-C.; Huh, J.; Reinertsen, J. F.; Ahtapodov, L.; Lee, K.; Heidari, B.; Van Helvoort, A., Position-controlled uniform GaAs nanowires on silicon using nanoimprint lithography. Nano letters 2014, 14 (2), 960-966.

17. Vukajlovic-Plestina, J.; Kim, W.; Dubrovski, V. G.; Tütüncüoğlu, G.; Lagier, M.; Potts, H.; Friedl, M.; Fontcuberta i Morral, A., Engineering the Size Distributions of Ordered GaAs Nanowires on Silicon. Nano Letters 2017, 17 (7), 4101-4108.

18. Bauer, B.; Rudolph, A.; Soda, M.; i Morral, A. F.; Zweck, J.; Schuh, D.; Reiger, E., Position controlled self-catalyzed growth of GaAs nanowires by molecular beam epitaxy. Nanotechnology 2010, 21 (43), 435601.

19. Russo-Averchi, E.; Vukajlovic Plestina, J.; Tütüncüoglu, G.; Matteini, F.; DalmauMallorquí, A.; de la Mata, M.; Rüffer, D.; Potts, H. A.; Arbiol, J.; Conesa-Boj, S.; Fontcuberta i Morral, A., High Yield of GaAs Nanowire Arrays on Si Mediated by the Pinning and Contact Angle of Ga. Nano Letters 2015, 15 (5), 2869-2874. 
20. Gibson, S.; LaPierre, R., Study of radial growth in patterned self-catalyzed GaAs nanowire arrays by gas source molecular beam epitaxy. physica status solidi (RRL) - Rapid Research Letters 2013, 7 (10), 845-849.

21. Dubrovskii, V.; Xu, T.; Álvarez, A. D.; Plissard, S.; Caroff, P.; Glas, F.; Grandidier, B., Self-equilibration of the diameter of Ga-catalyzed GaAs nanowires. Nano letters 2015, 15 (8), 5580-5584.

22. Persson, A.; Ohlsson, B.; Jeppesen, S.; Samuelson, L., Growth mechanisms for GaAs nanowires grown in CBE. J. Cryst. Growth 2004, 272 (1), 167-174.

23. García Núñez, C.; Braña, A. F.; López, N.; García, B. J., GaAs Nanowires Grown by Gaassisted Chemical Beam Epitaxy: Substrate Preparation and Growth Kinetics. J. Cryst. Growth 2015, 430, 108-115.

24. García Núñez, C.; Braña, A. F.; Pau, J. L.; Ghita, D.; García, B. J.; Shen, G.; Wilbert, D. S.; Kim, S. M.; Kung, P., Pure zincblende GaAs nanowires grown by Ga-assisted chemical beam epitaxy. Journal of Crystal Growth 2013, 372, 205-212.

25. García Núñez, C.; Braña, A. F.; Pau, J. L.; Ghita, D.; García, B. J.; Shen, G.; Wilbert, D. S.; Kim, S. M.; Kung, P., Surface optical phonons in GaAs nanowires grown by Ga-assisted chemical beam epitaxy. Journal of Applied Physics 2014, 115 (3), 034307.

26. Perea, D. E.; Allen, J. E.; May, S. J.; Wessels, B. W.; Seidman, D. N.; Lauhon, L. J., Threedimensional nanoscale composition mapping of semiconductor nanowires. Nano letters 2006, 6 (2), 181-185.

27. Dubrovskii, V.; Cirlin, G.; Sibirev, N.; Jabeen, F.; Harmand, J.; Werner, P., New mode of vapor- liquid- solid nanowire growth. Nano letters 2011, 11 (3), 1247-1253.

28. Matteini, F.; Dubrovskii, V. G.; Rüffer, D.; Tütüncüoğlu, G.; Fontana, Y.; Morral, A. F. I., Tailoring the diameter and density of self-catalyzed GaAs nanowires on silicon. Nanotechnology 2015, 26 (10), 105603.

29. Fontcuberta i Morral, A.; Colombo, C.; Abstreiter, G.; Arbiol, J.; Morante, J., Nucleation mechanism of gallium-assisted molecular beam epitaxy growth of gallium arsenide nanowires. Applied Physics Letters 2008, 92 (6), 063112-063112-3.

30. Matteini, F.; Tutuncuoglu, G.; Rüffer, D.; Alarcon-Llado, E.; Morral, A., Untangling the role of oxide in Ga-assisted growth of GaAs nanowires on Si substrates. arXiv preprint arXiv:1307.6113 2013.

31. Colombo, C.; Spirkoska, D.; Frimmer, M.; Abstreiter, G.; i Morral, A. F., Ga-assisted catalyst-free growth mechanism of GaAs nanowires by molecular beam epitaxy. Physical Review $B$ 2008, 77 (15), 155326.

32. Dayeh, S. A.; Soci, C.; Bao, X.-Y.; Wang, D., Advances in the synthesis of InAs and GaAs nanowires for electronic applications. Nano Today 2009, 4 (4), 347-358.

33. Jensen, L. E.; Björk, M. T.; Jeppesen, S.; Persson, A. I.; Ohlsson, B. J.; Samuelson, L., Role of surface diffusion in chemical beam epitaxy of InAs nanowires. Nano Letters 2004, 4 (10), 1961-1964.

34. Dubrovskii, V.; Sibirev, N.; Cirlin, G.; Soshnikov, I.; Chen, W.; Larde, R.; Cadel, E.; Pareige, P.; Xu, T.; Grandidier, B., Gibbs-Thomson and diffusion-induced contributions to the growth rate of Si, InP, and GaAs nanowires. Physical Review B 2009, 79 (20), 205316.

35. García Núñez, C.; Braña, A. F.; López, N.; Pau, J. L.; García, B. J. In Photodetector fabrication by dielectrophoretic assembly of GaAs nanowires grown by a two-steps method, SPIE Nanoscience + Engineering, San Diego, SPIE: San Diego, 2017; p 103530F. 
TOC Figure

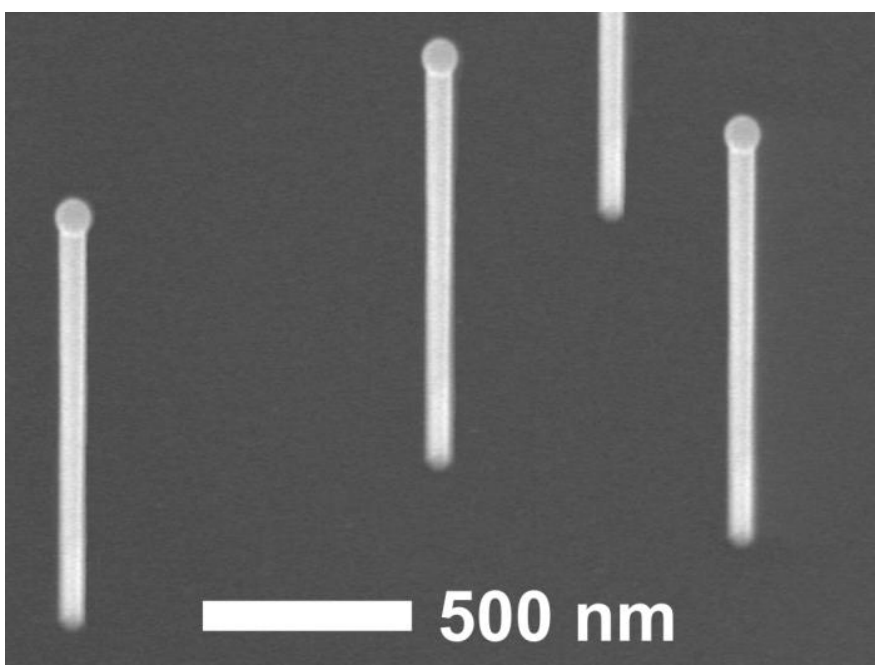

